\title{
Hybridbildung bei der Gattung Echium (Boraginaceae) auf Tenerife, Kanarische Inseln \\ Marcus Werner
}

\begin{abstract}
Two hybrids recently found on Tenerife (Canary Islands), Echium wildpretii $\mathrm{x}$ virescens and Echium simplex $\mathrm{x}$ strictum, are presented. Their characteristics are compared with those of the parental taxa. Descriptions of their habitats and a comment on other Echium hybrids are provided.
\end{abstract}

\section{Zusammenfassung}

Die zwei unlängst auf der Kanareninsel Tenerife gefundenen Hybriden Echium wildpretii x virescens und Echium simplex x strictum werden vorgestellt und ihre charakteristischen Merkmale mit denen der Ausgangssippen verglichen. Ferner erfolgt eine Standortbeschreibung und der Hinweis auf weitere Hybriden innerhalb der Gattung Echium.

\section{Resumen}

En el artículo se presentan los dos híbridos Echium wildpretii $\mathrm{x}$ virescens y Echium simplex $\mathrm{x}$ strictum, que hace poco fueron encontrados en la Isla Canaria de Tenerife. Del mismo modo se comparan sus características distintivas con las de las especies de partida. Además aparecen descripciones del hábitat y observaciones sobre otros híbridos del género Echium.

\section{Einführung}

Die Kanarischen Inseln bieten bekanntlich gute Rahmenbedingungen für Artbildungsprozesse, die sich im Laufe der Zeit vor allem durch adaptive Radiation vollzogen haben.

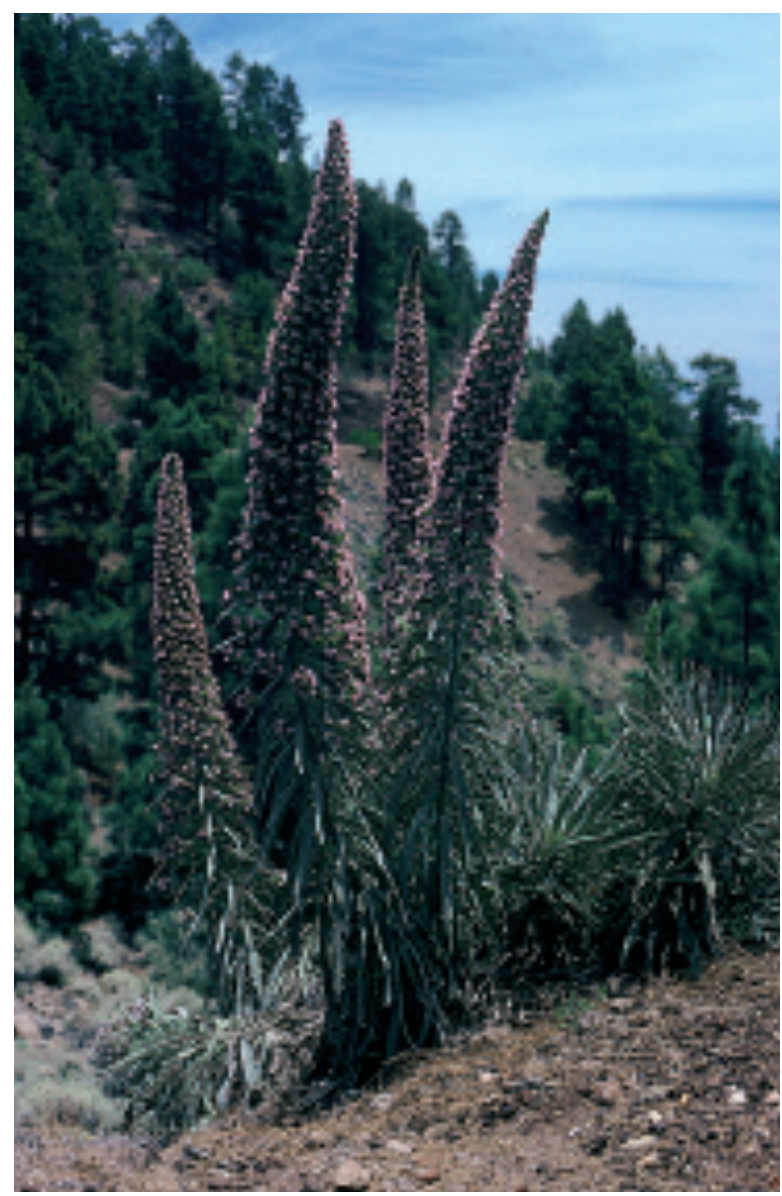

Ob auch natürliche Hybridisierungsvorgänge für die Evolution einzelner Sippen eine Rolle spielen konnten, ist bezüglich der Kanarischen Inseln bisher kaum untersucht worden. Gleichwohl wurden besonders innerhalb der weitgehend endemischen Crassulaceen-Gattungen Aeonium, Aichryson, Greenovia und Monanthes zahlreiche Hybriden - auch zwischen verschiedenen Gattungen - beschrieben (BAÑARES BAUDET 1986, 1990, 1996). Hybriden sind aber auch unter den endemischen Arten der Gattungen Sonchus, Micromeria, Lavandula, Sideritis, Bystropogon und Euphorbia bekannt (vgl. HANSEN \& Sunding 1993, Hohenester \& Welss 1993). Dass Hybridisierungsvorgänge aber noch weiter gehen, soll hier am Beispiel der Gattung Echium dargestellt werden.

Auf der Kanareninsel Tenerife konnte im April 2000 eine Population von Pflanzen gefunden werden, die sich eindeutig als Hybriden zwischen den endemischen Natternkopf-Arten Echium wildpretii und E. virescens identifizieren ließen (vgl. Abb. 1). Der Standort befand sich oberhalb von Vilaflor unweit der

Abb. 1: Echium wildpretii $\mathrm{x}$ virescens nahe der Piste zur Paisaje Lunar. 
Piste, die in die sogenannte Paisaje Lunar führt. Dort kommen außer den Hybriden auch die Elternarten zusammen vor. Vor allem Echium virescens bildet hier prächtige Bestände.

\section{Die Hybriden und ihre Elternarten}

\subsection{Echium wildpretii $x$ virescens}

Es handelt sich um eine Hybride zwischen Echium virescens DC. (var. angustissimum Bolle et Christ) und E. wildpretii H. W. PEARson et HoOK. f. ssp. wildpretii.
Fundort: $5 \mathrm{~km}$ auf der Piste oberhalb Vilaflor (im Süden von Tenerife) in Richtung Paisaje Lunar, ca. $1680 \mathrm{~m}$ ü. d. M., in einer scharfen Kurve, die einen Barranco durchquert, unterhalb der Piste in den relativ steilen Hängen (besonders am ostexponierten Hang)

Aufgrund ihrer Wuchs- (vgl. Abb. 2) und Blütenstandsform (vgl. Abb. 1) sowie der Blütenfarbe (vgl. Abb. 3) lassen sich die Pflanzen leicht und eindeutig als Hybriden der erwähnten Elternarten identifizieren (siehe

\begin{tabular}{|c|c|c|c|}
\hline & E. virescens & $\begin{array}{l}\text { E. wildpretii } \mathbf{x} \\
\text { virescens }\end{array}$ & E. wildpretii \\
\hline Lebensform & $\begin{array}{l}\text { reich verzweigter } \\
\text { Strauch, bis } 2,5 \mathrm{~m} \text {; } \\
\text { Seitenzweige meist } \\
\text { um } 6 \mathrm{~cm} \text { im Durch- } \\
\text { messer }\end{array}$ & $\begin{array}{l}\text { basal verzweigter } \\
\text { Strauch, bis } 1,5 \mathrm{~m} \text {; } \\
\text { Seitenzweige bis } 4 \mathrm{~cm} \\
\text { im Durchmesser }\end{array}$ & $\begin{array}{l}\text { Halbrosettenpflanze; } \\
\text { bis } 0,5 \mathrm{~m} \text {, mit kurzem, } \\
\text { verholztem Stamm, } \\
\text { manchmal basal } \\
\text { verzweigt; Seiten- } \\
\text { zweige bis } 4 \mathrm{~cm} \text { im } \\
\text { Durchmesser }\end{array}$ \\
\hline Blattstellung & $\begin{array}{l}\text { an den Triebenden } \\
\text { gehäuft, nicht } \\
\text { rosettig; } \\
\text { „Federbuschstrauch“ }\end{array}$ & $\begin{array}{l}\text { keine echten } \\
\text { Rosetten, obwohl } \\
\text { an den Triebenden } \\
\text { gehäuft }\end{array}$ & $\begin{array}{l}\text { dichte Blattrosetten } \\
\text { an den Triebenden }\end{array}$ \\
\hline Blattform & $\begin{array}{l}\text { lineal bis lanzettlich, } \\
\text { kurz gestielt }\end{array}$ & $\begin{array}{l}\text { lineal bis lineal- } \\
\text { lanzettlich, deutlich } \\
\text { gestielt }\end{array}$ & $\begin{array}{l}\text { lineal bis lineal- } \\
\text { lanzettlich, deutlich } \\
\text { gestielt }\end{array}$ \\
\hline Behaarung & seidig-borstig & seidig-borstig & lang seidig-borstig \\
\hline Blütenstand & $\begin{array}{l}10 \text { bis } 30 \mathrm{~cm} \text {, } \\
\text { zylindrisch bis } \\
\text { kerzenförmig, } \\
\text { sehr dicht }\end{array}$ & $\begin{array}{l}0,5 \text { bis } 1,2 \mathrm{~m} \text {; } \\
\text { kegelförmig, etwas } \\
\text { aufgelockert }\end{array}$ & $\begin{array}{l}1,5 \text { bis } 3 \mathrm{~m} \text {, } \\
\text { zylindrisch, unterhalb } \\
\text { der Mitte am breites- } \\
\text { ten, sehr dicht }\end{array}$ \\
\hline Blütenfarbe & rosa, selten blassblau & $\begin{array}{l}\text { rosa, beim Verblühen } \\
\text { blassblau }\end{array}$ & rot, verblüht blau \\
\hline Blütenkrone & schmal trichterförmig & weit trichterförmig & weit trichterförmig \\
\hline $\begin{array}{l}\text { Farbe der Staub- } \\
\text { blätter und des } \\
\text { Griffels }\end{array}$ & rosa bis rötlich & $\begin{array}{l}\text { rosa, Staubbeutel } \\
\text { blau }\end{array}$ & $\begin{array}{l}\text { rötlich, Staubbeutel } \\
\text { blau }\end{array}$ \\
\hline
\end{tabular}

Tab. 1: Vergleich von Echium wildpretii x virescens mit den Elternarten 
Tab.1).

Die Hybridpflanzen blühen am Standort zur selben Zeit wie Echium wildpretii, während dort Echium virescens schon verblüht ist. Die Vegetation des Standortes lässt sich dem trockenen Kiefernwald der Südseite zuordnen. Bei Vegetationsaufnahmen konnten folgende Arten notiert werden: Sideritis soluta ssp. soluta, Argyranthemum adauctum ssp. duguorii, Pinus canariensis, Chamaecytisus proliferus, Carlina xeranthemoides, Bystropogon origanifolius und Lotus campylocladus. Als weitere Begleiter wurden in den Aufnahmeflächen Echium virescens, Echium wildpretii, Pterocephalus lasiospermus, Tolpis webbii, Silene vulgaris ssp. commutata, Avena barbata, Phagnalon saxatile, Bromus rigidus, Bromus tectorum und Aeonium smithii festgestellt.

\subsection{Echium simplex $\mathrm{x}$ E. strictum $\mathrm{ssp}$. strictum} Es handelt sich um eine Hybride zwischen Echium simplex DC. und Echium strictum L. f. ssp. strictum. Fundort: Nordabfall des Anaga-Gebirges, am Rand des Wanderweges bei Las Breñas zwischen Las Palmas und Faro de Anaga; 170 m ü. d. M.; arten- und endemitenreicher Sukkulentenbusch der xerophytischen Zone. Auch hier kommen die beiden Elternarten zusammen in der Nähe des

\begin{tabular}{|c|c|c|c|}
\hline & E. simplex & $\begin{array}{l}\text { E. simplex } \mathbf{x} \\
\text { E. strictum }\end{array}$ & E. strictum \\
\hline Lebensform & $\begin{array}{l}\text { Halbrosettenpflanze } \\
\text { mit kurzem, ver- } \\
\text { holztem Stamm (mit } \\
\text { Blütenstand bis } 2 \mathrm{~m} \text { ) }\end{array}$ & $\begin{array}{l}\text { Halbrosettenpflanze } \\
\text { (mit Blütenstand } \\
\text { bis } 1 \mathrm{~m} \text { ); mit kurzem, } \\
\text { verholztem Stamm }\end{array}$ & $\begin{array}{l}\text { kleiner verzweigter } \\
\text { Strauch, bis } 1 \mathrm{~m}\end{array}$ \\
\hline Blattstellung & $\begin{array}{l}\text { Blätter in einer } \\
\text { dichten Rosette am } \\
\text { Stammende }\end{array}$ & $\begin{array}{l}\text { Blätter in einer } \\
\text { dichten Rosette am } \\
\text { Stammende }\end{array}$ & $\begin{array}{l}\text { Blätter an den } \\
\text { Triebenden gehäuft, } \\
\text { aber nicht rosettig }\end{array}$ \\
\hline Blattform & $\begin{array}{l}\text { eiförmig bis elliptisch, } \\
\text { blaugrün, Blattstiel } \\
\text { kurz am Grund } \\
\text { verbreitert }\end{array}$ & $\begin{array}{l}\text { oval-eiförmig, dünn; } \\
\text { Blattstiel kurz, am } \\
\text { Grund wenig } \\
\text { verbreitert }\end{array}$ & $\begin{array}{l}\text { oval-eiförmig, dünn; } \\
\text { Blattstiel kurz, am } \\
\text { Grund wenig } \\
\text { verbreitert }\end{array}$ \\
\hline Behaarung & seidig-borstig & steif-borstig & steif-borstig \\
\hline Blütenstand & $\begin{array}{l}\text { zylindrisch, bis } 2 \mathrm{~m} \text {, } \\
\text { dicht }\end{array}$ & $\begin{array}{l}\text { zylindrisch, bis } 60 \mathrm{~cm} \text {, } \\
\text { locker }\end{array}$ & $\begin{array}{l}\text { bis } 30 \mathrm{~cm} \text {, lockerer } \\
\text { Thyrsus }\end{array}$ \\
\hline Blütenfarbe & $\begin{array}{l}\text { weiß, mit blassblauer } \\
\text { Aderung }\end{array}$ & hellrosa & blassblau \\
\hline Blütenkrone & schmal glockenförmig & trichterförmig & trichterförmig \\
\hline $\begin{array}{l}\text { Farbe der Staub- } \\
\text { blätter und des } \\
\text { Griffels }\end{array}$ & weiß & hellrosa & rosa \\
\hline
\end{tabular}

Tab. 2: Merkmale von Echium simplex, E. strictum und deren Hybride 


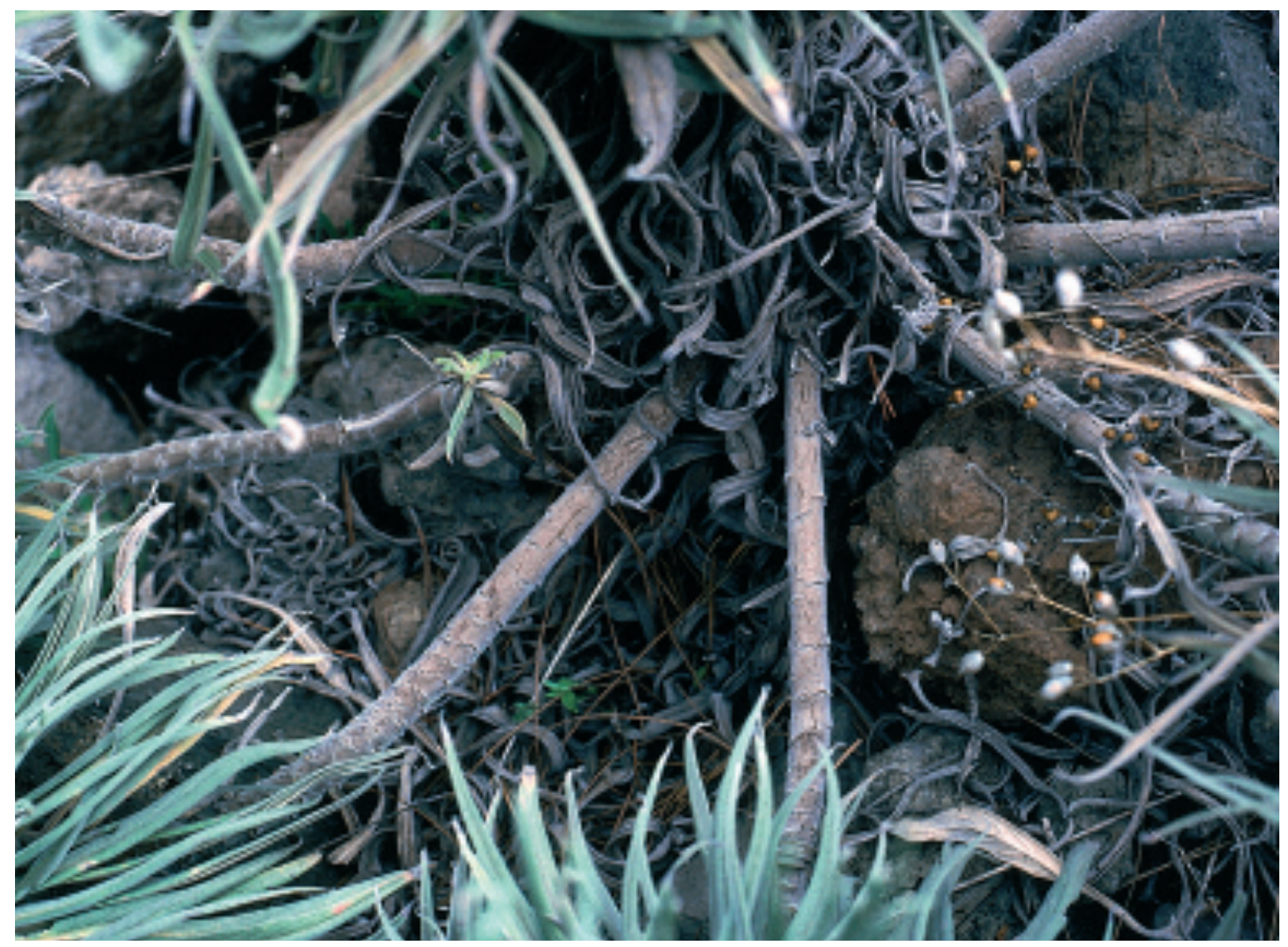

Standortes der Hybriden vor (vgl. Abb. 4).

2.3 Weitere Hybriden der Gattung Echium auf den Kanaren

Auf El Hierro kommt Echium x taibiquense vor (WolfF \& Rosinski 1999), eine Hybride der Arten E. aculeatum und E. hierrense. SCHÖNFELDER et al. (1993) geben für Tenerife das Vorkommen von E. auberianum x E. wildpretii an. Vielleicht handelt es sich bei dem Individuum in Abb. 5 um diese Hybride. Weniger eindeutig zu bestimmen ist eine Population von Sträuchern im Teno-Gebirge, die eventuell Hybriden der Arten E. virescens und E. aculeatum darstellen können. Habitus sowie Form und Struktur der Blätter erinnern an E.virescens, die Gestalt des Blütenstandes und die Farbe der Blüten jedoch eher an

Abb. 2 (oben): Wuchsform von Echium wildpretii $\mathrm{x}$ virescens.

Abb. 3 (unten): Blütenstand von Echium wildpretii $\mathrm{x}$ virescens.

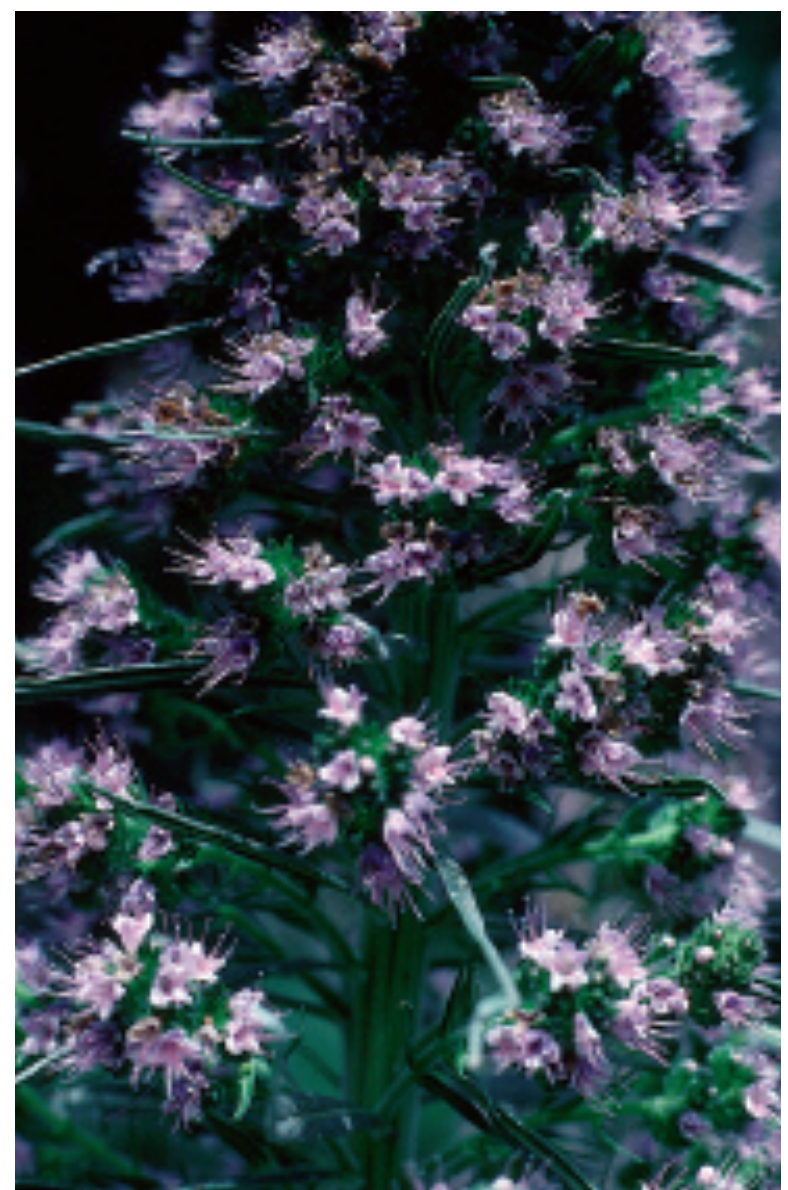




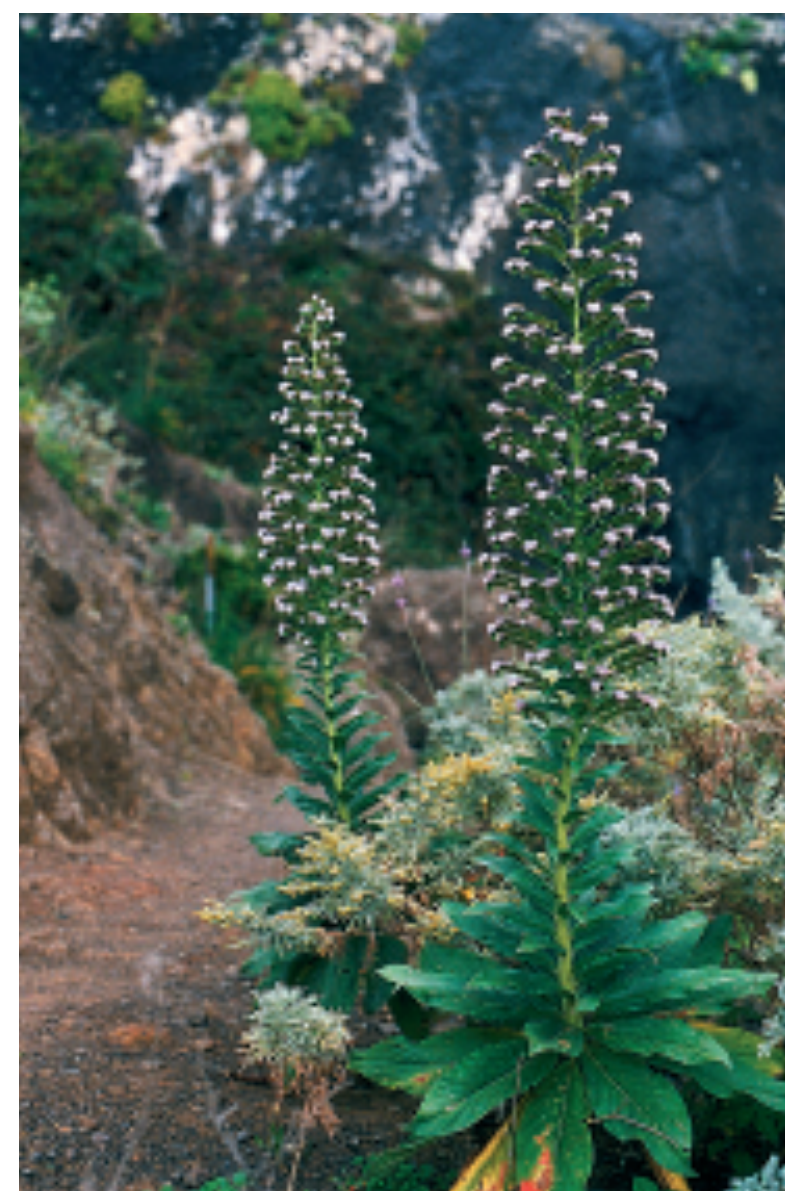

\section{E. aculeatum.}

\section{Diskussion}

Die durch adaptive Radiation in einem relativ kurzen Zeitraum entstandenen, endemischen Arten der Gattung Echium sind, ungeachtet der morphologischen Vielfalt, nur unvollstän-

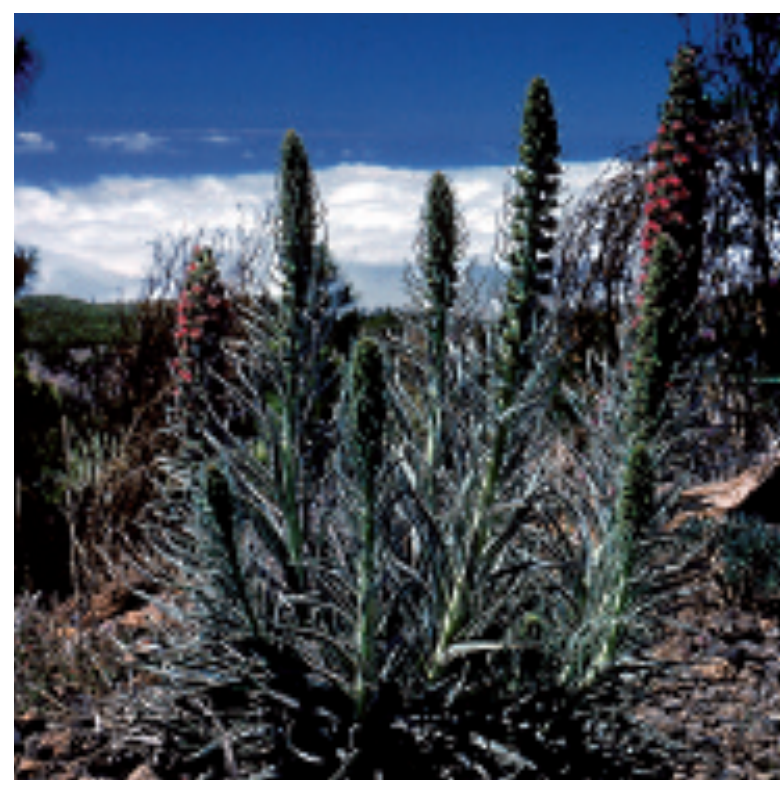

dig voneinander isoliert. Inzwischen ist von vielen Gattungen ozeanischer Inseln bekannt, dass deren Arten miteinander kreuzbar sind. Alle endemischen Echium-Arten Tenerifes sind diploid $(2 \mathrm{n}=16)$, was Hybridisierung grundsätzlich erleichtert. Des weiteren berühren oder überschneiden sich die Areale vieler Arten, obwohl sie an unterschiedliche ökologische Nischen angepasst sind.

Bis vor kurzem nahm man an, dass Hybridisierungen in der natürlichen Umwelt gewissermaßen „Unfälle“ der Fortpflanzung darstellen. Den so genannten „Bastarden“ wird meist nur eine geringe Lebens- und Fortpflanzungsfähigkeit zugesprochen. Doch seit einigen Jahren hält man es durchaus für möglich, dass auch durch Hybridisierung und Introgression neue Arten entstehen können (z. B. ARNOLD 1997). Dies konnte auch mehrfach bestätigt werden, auf Tenerife z. B. von Brochmann et al. (2000) im Fall des endemischen Korbblütlers Argyranthemum sundingii.

Damit Hybridpopulationen sich erfolgreich etablieren können, müssen sie sowohl der Möglichkeit von Rückkreuzungen als auch der Konkurrenz mit den Ausgangssippen ausweichen, indem sie neue Lebensräume erschließen. Sie benötigen darüber hinaus ein notwendiges $\mathrm{Ma}$ an Fortpflanzungsfähigkeit und Stabilität.

Erschwerend für das Studium der Natternkopf-Hybriden auf Tenerife ist, dass es sich fast ausschließlich um ausdauernde Pflanzen handelt, die erst nach einigen Jahren das erste Mal zur Blüte kommen. Wichtig ist nämlich die Beobachtung der Hybriden über mehrere Generationen hinweg, um feststellen zu können, ob sie fertil oder steril sind. Die meisten dieser

Abb. 4 (oben): Echium simplex x Echium strictum ssp. strictum im Anaga-Gebirge.

Abb. 5 (unten): Echium wildpretii $\mathrm{x}$ auberianum?

Abb. 6(S. 157): Freigelegtes Exemplar von Echium wildpretii, Blattrosette von unten. Ältere, abgestorbene Blätter wurden entfernt, um die kurzen, verholzten Seitenzweige sichtbar zu machen. 


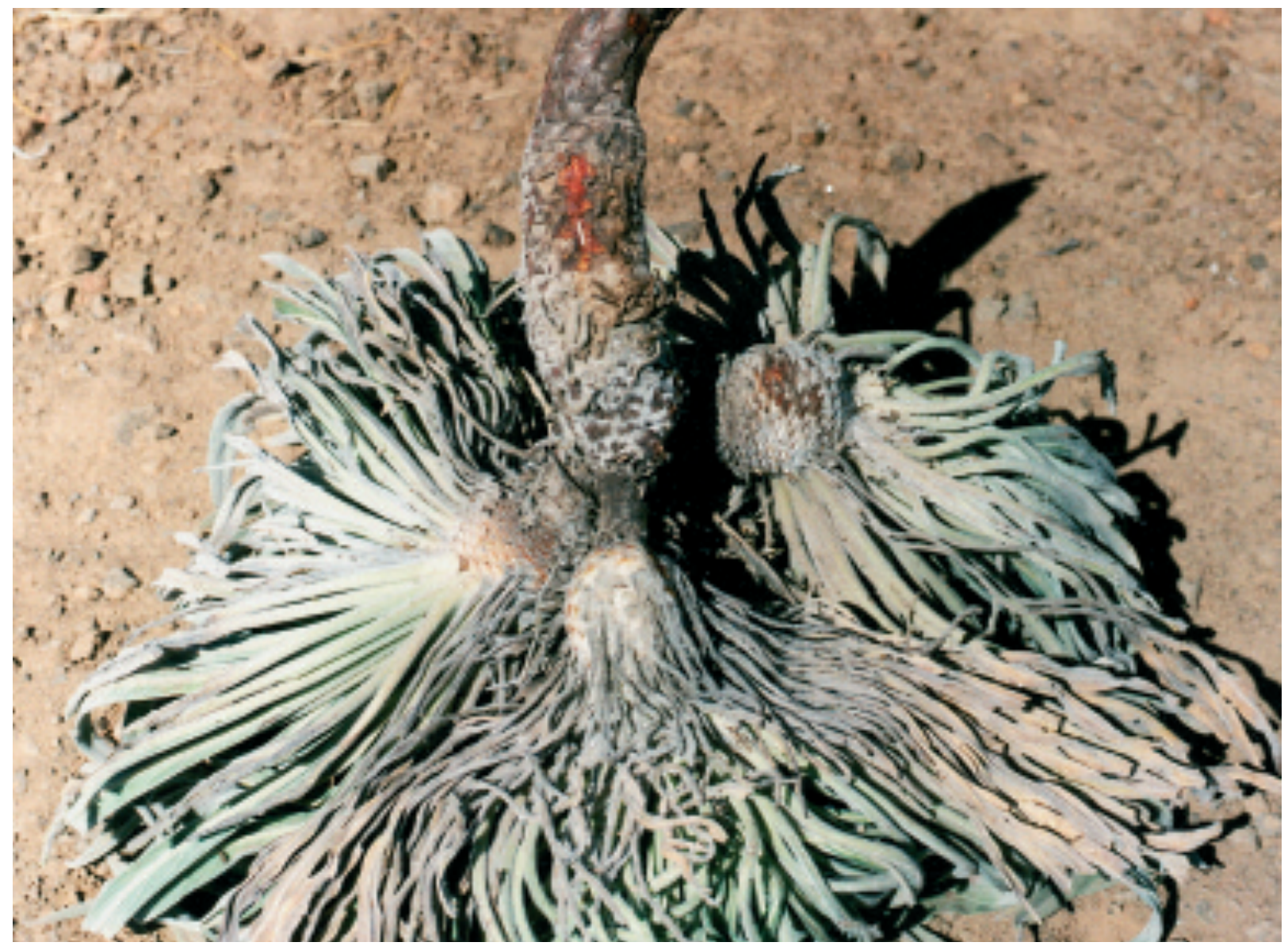

Hybrid-Populationen erlöschen nach einiger Zeit wieder, können aber an anderer oder gleicher Stelle erneut entstehen. Artbildung durch Hybridisierung innerhalb der Gattung Echium auf Tenerife ist sicher sehr unwahrscheinlich, aber prinzipiell auch nicht auszuschließen.

\section{Dank}

Die Arbeit ist im Zusammenhang mit standortökologischen Untersuchungen auf der Insel Tenerife entstanden, die von Mitarbeitern der Arbeitsgruppe Prof. Dr. DIETER LÜPNITZ (Universität Mainz) durchgeführt und seitens der Feldbauschstiftung freundlicherweise unterstützt wurde. Die hier wiedergegebenen Resultate sind als Teilergebnis umfangreicherer Untersuchungen an Echium-Standorten zu sehen.

\section{Literatur}

ARNOLD, M. L. 1997: Natural hybridzation and evolution.

- Oxford.

BAÑARES BAUdeT, Á. 1986: Híbridos interespecificos del genero Aeonium WeBB et BERTH. (Crassulaceae) en las islas Canarias. Novedades y datos corológicos. - Vieraea 16: 57-71.

Bañares BAudet, Á. 1990: Híbridos de la familia Crassulaceae en las islas Canarias. Novedades y datos corológicos. II. - Vieraea 18: 65-85.

BAÑAREs BAudeT, Á. 1996: Híbridos de la familia Crassulaceae en las islas Canarias. Novedades y datos corológicos. III. - Vieraea 25: 181-195.

Bramwell, D. 1972: A revision of the genus Echium in Macaronesia. - Lagascalia 2: 237-115.

Brochmann, C., Borgen, L.\& Stabbetorp, O. E. 2000: Multiple diploid hybrid speciation of the Canary Island endemic Argyranthemum sundingii (Asteraceae). - Plant. Syst. Evol. 220: 77-92.

Crawford, D. J. \& Stuessy, T. F. 1997: Plant speciation on oceanic islands. In: K. IwATSUKI \& RAVEN, P. H. (Hrsg.): Evolution and diversification of land plants. - Tokyo.

Hansen, A. \& Sunding, P. 1993: Flora of Macaronesia. Checklist of vascular plants. - Sommerfeltia 17.

HohEnester, A. \& Welss, W. 1993: Exkursionsflora für die Kanarischen Inseln. - Stuttgart.

SChÖNFElder, P., LeÓn Arencibia, M.C. \& Wildpret de LA TORRE, W. 1993: Catálogo de la flora vascular de la Isla 